\title{
Influence of trait anxiety, child maltreatment, and adulthood life events on depressive symptoms
}

This article was published in the following Dove Press journal:

Neuropsychiatric Disease and Treatment

\author{
Yoshihiro Uchida ${ }^{1-3}$ \\ Toshinao Takahashi ${ }^{1,3}$ \\ Shigemasa Katayama ${ }^{1,3}$ \\ Jiro Masuya ${ }^{1,2}$ \\ Masahiko Ichiki' \\ Hajime Tanabe ${ }^{4}$ \\ Ichiro Kusumi ${ }^{5}$ \\ Takeshi Inoue' \\ 'Department of Psychiatry, Tokyo \\ Medical University, Shinjuku-ku, Tokyo \\ 160-0023, Japan; ${ }^{2}$ Department of \\ Psychiatry, Ibaraki Medical Center, \\ Tokyo Medical University, Ami- \\ machi, Inashiki-gun, Ibaraki 300-0395, \\ Japan; ${ }^{3}$ Department of Psychiatry, \\ Seijin Hopital, Adachi-ku, Tokyo \\ 121-08/5, Japan; ${ }^{4}$ Department of \\ Clinical Human Sciences, Graduate \\ School of Humanities and Social \\ Sciences, Shizuoka University, \\ Suruga-ku, Shizuoka 422-8529, Japan; \\ ${ }^{5}$ Department of Psychiatry, Hokkaido \\ University Graduate School of \\ Medicine, Kita-ku, Sapporo 060-8638, \\ Japan
}

Correspondence: Takeshi Inoue Department of Psychiatry, Tokyo Medical University, 6-7-I, Nishishinjuku, Shinjuku-ku, Tokyo 160-0023, Japan

Tel +8 I 333426 III ext 5754

Fax +8I 333404499

Email tinoue@tokyo-med.ac.jp
Background: Various personality traits mediate the association between childhood stress and depressive symptoms in adulthood. The aim of this study was to clarify the indirect effects of the experience of child maltreatment on depressive symptoms and appraisal of life events in adulthood through trait anxiety.

Subjects and methods: A total of 404 participants who were volunteer subjects from the community were studied using the following self-administered questionnaire surveys: Patient Health Questionnaire-9, which measures depressive symptoms; State-Trait Anxiety Inventory Form Y, which measures trait anxiety; the Child Abuse and Trauma Scale, which measures child maltreatment; and Life Experiences Survey, which measures negative and positive appraisal of adulthood life events.

Results: Structural equation modeling demonstrated that the experience of child maltreatment increased depressive symptoms in adulthood as well as the negative appraisal of life events in adulthood through an increase in trait anxiety. Furthermore, trait anxiety affected depressive symptoms in adulthood through its influence on the negative appraisal of adulthood life events. The following indirect effect was also significant: the experience of child maltreatment increased the negative appraisal of adulthood life events via trait anxiety and subsequently influenced adult depressive symptoms.

Limitations: The subjects of this study are volunteer subjects from the community including healthy people, and hence the results may not be generalizable to major depressive patients. Recall bias should be considered when interpreting the results. Because this study is a crosssectional study, the causality between the experience of child maltreatment and depression is not clear.

Conclusion: This study suggests that trait anxiety may play a mediating role in the influence of the experience of child maltreatment on depressive symptoms in adulthood and negative appraisal of adulthood life events.

Keywords: child maltreatment, covariance structure analysis, depression, STAI-Y, trait anxiety, life events

\section{Introduction}

Major depressive disorder (MDD) is a common psychiatric disorder, with a lifetime prevalence of $5 \%-17 \%$ of the general population. ${ }^{1}$ The most recent Global Burden of Disease Study reported that MDD is the second leading cause of years lived with disability. ${ }^{2}$ MDD is caused by the interaction of multiple factors, such as heredity, personality traits, experience of child maltreatment, parenting, and stressful adulthood life events. ${ }^{3-5}$ Previous studies pointed out that persons who were abused in childhood have a higher incidence of major depressive episodes in adulthood, ${ }^{4}$ and patients with depression experienced more harmful events in childhood than healthy controls. ${ }^{6}$ 
Because there is a long time between the experience of child stress and MDD in adulthood, it is assumed that there are multiple mediators between them.

Previously, we reported that affective temperament, a personality trait associated with depression, plays a major role in the effect of the experience of child maltreatment on adulthood depressive symptoms, as both a moderator and a mediator, in adult volunteer subjects from the community. ${ }^{7,8}$ In addition, affective temperament plays roles in the effects of the experience of child maltreatment on depressive symptoms and is a mediator of depressive symptoms in MDD patients..$^{9,10}$ Neuroticism, which is another temperament, has been established as a risk factor for developing depression. High levels of neuroticism tend to induce the development of depressive episodes in response to stressful life events. ${ }^{5}$ The quality of parenting, mediated by neuroticism, affects the severity of depressive symptoms in patients with MDD. ${ }^{11}$ Our previous study indicated that neuroticism is also a mediator for the effects of the experience of child maltreatment and quality of parenting on adulthood depressive symptoms, as well as the negative appraisal of life events in volunteer subjects from the community. ${ }^{12,13}$ Therefore, several personality traits have been proposed as mediators for the effect of childhood stress on depression.

On the other hand, it is known that "trait anxiety" constitutes a public mental health problem. ${ }^{14} \mathrm{~A}$ higher therapeutic response in depressive disorders is associated with increased age, longer treatment duration, higher resilience, and lower trait anxiety. ${ }^{15}$ In addition, trait anxiety has been implicated as a vulnerability characteristic associated with the development of stress-induced depression. ${ }^{16}$ Furthermore, in cardiac surgery patients, trait anxiety mediates the effect of stress exposure on postoperative posttraumatic stress disorder and depression. ${ }^{17}$ Although trait anxiety is a personality trait that makes people more vulnerable to depression, it remains unclear whether trait anxiety is involved as a mediator in the effects of the experience of child maltreatment on adulthood depressive symptoms.

Therefore, the aim of this study was to clarify how three factors, namely, trait anxiety, adulthood life events, and the experience of child maltreatment, are associated with adulthood depressive symptoms in adult volunteer subjects from the community and whether the experience of child maltreatment has an indirect effect on depressive symptoms in adulthood and the appraisal of adulthood life events through trait anxiety. Consequently, we analyzed trait anxiety evaluated using the State-Trait Anxiety Inventory Form Y (STAI-Y), ${ }^{18}$ child maltreatment measured using the
Child Abuse and Trauma Scale (CATS), ${ }^{19}$ adulthood life events assessed using the Life Experiences Survey (LES), ${ }^{20}$ and demographic characteristics in volunteer subjects from the community, and analyzed the association between the above three causative factors and depressive symptoms by structural equation modeling (SEM).

\section{Subjects and methods Subjects}

This research was part of a larger study, conducted from January to August 2014. ${ }^{12-14}$ We recruited volunteers by flyers and word of mouth and distributed the self-administered questionnaires to 853 Japanese adult volunteers. Of the 853 volunteers, 404 subjects $(47.4 \%$; 220 men, 184 women; age $42.3 \pm 11.9$ years) agreed to participate in this study and provided complete responses to the questionnaires.

Subjects completed the self-administered questionnaire anonymously. Written informed consent was obtained from all of the subjects after giving the following explanations: 1) participation in this research and replies to the questions can be freely decided by individual judgment; 2) if they do not agree to participate in this research, they will not experience any disadvantages; 3) data of this research will be managed by making all data anonymous in a format which cannot identify individuals; and 4) personal information will not be leaked to the outside. In accordance with the Declaration of Helsinki, this study was conducted with approval from the ethics committee of Tokyo Medical University and Hokkaido University Hospital.

\section{Questionnaires}

\section{Patient Health Questionnaire-9 (PHQ-9)}

Spitzer et $\mathrm{al}^{21}$ developed the PHQ-9, which is a self-administered questionnaire for the screening of major depressive episodes and the evaluation of the severity of depressive symptoms. The Japanese version was developed and its validity was substantiated by Muramatsu et al. ${ }^{22}$ Major depressive episodes were diagnosed in two ways, by applying the PHQ-9 diagnostic algorithms and a summary score. ${ }^{23}$ This study used a summary score for evaluating the severity of depressive symptoms. Specifically, the total score (0-27 points) of nine items of depressive symptoms in the previous 2 weeks using a 4-point Likert scale for each item were calculated $(0-3,0=$ not at all, $1=$ several days, $2=$ more than half the days, and $3=$ nearly every day).

\section{State-Trait Anxiety Inventory Form $Y$}

The STAI-Y is a 40-item self-reported measure of transient (state) and chronic (trait) anxiety symptoms. ${ }^{18}$ The STAI-Y state anxiety section consists of 20 statements by which 
respondents assess how they feel on a 4-point scale ( $1=$ not at all, $2=$ somewhat, $3=$ moderately so, and $4=$ very much so) for each statement. The STAI-Y trait anxiety section consists of 20 statements by which respondents evaluate how they feel on a 4 -point scale ( $1=$ almost never, $2=$ sometimes, $3=$ often, and $4=$ almost always). The STAI-Y state and trait scores range from 20 to 80 .

\section{Child Abuse and Trauma Scale}

The CATS is a 38-item self-administered questionnaire to retrospectively evaluate adverse childhood experiences. ${ }^{19}$ Tanabe, an author of the present manuscript, developed and validated the Japanese version of the CATS using the classical translation-back translation technique with the permission and confirmation of Dr Sanders. ${ }^{24}$ Participants evaluate how frequently they had a particular abusive experience during their childhood and adolescence, using a 5 -point scale $(0=$ never, $1=$ rarely, $2=$ sometimes, $3=$ very often, and $4=$ always). There are three subscales, measuring the subjective reports of the following three aspects of adverse childhood experiences: neglect/negative home atmosphere, punishment, and sexual abuse. In the present study, the average score of 38 items were calculated as the total score, and the average scores of each subscale were also calculated.

\section{Life Experiences Survey}

The LES is a 57-item self-administered questionnaire. The respondents state the major events that they experienced in the previous year. ${ }^{20}$ The Japanese version of the LES was used in this study. ${ }^{7}$ The respondents evaluated the intensity of the psychological impact of the event using a 7-point scale, ranging from extremely negative $(-3)$ to extremely positive $(+3)$. The positive change score summing the effect of positive events and the negative change score summing the effect of negative events were calculated.

\section{Construction of the structural equation model}

A structural equation model was constructed using the PHQ-9 scores, STAI-Y trait anxiety scores, LES positive change scores, LES negative change scores, and CATS subscale scores (Figure 1). The average subscale scores of the CATS (sexual abuse, neglect, and punishment) were the observed variables in the CATS, and the latent variable "child maltreatment" was comprised of these subscales. In this model, the experience of child maltreatment, trait anxiety, LES positive and negative change scores (positive and negative appraisal of life events) were considered to directly affect depressive symptoms in adulthood. Furthermore, the experience of child

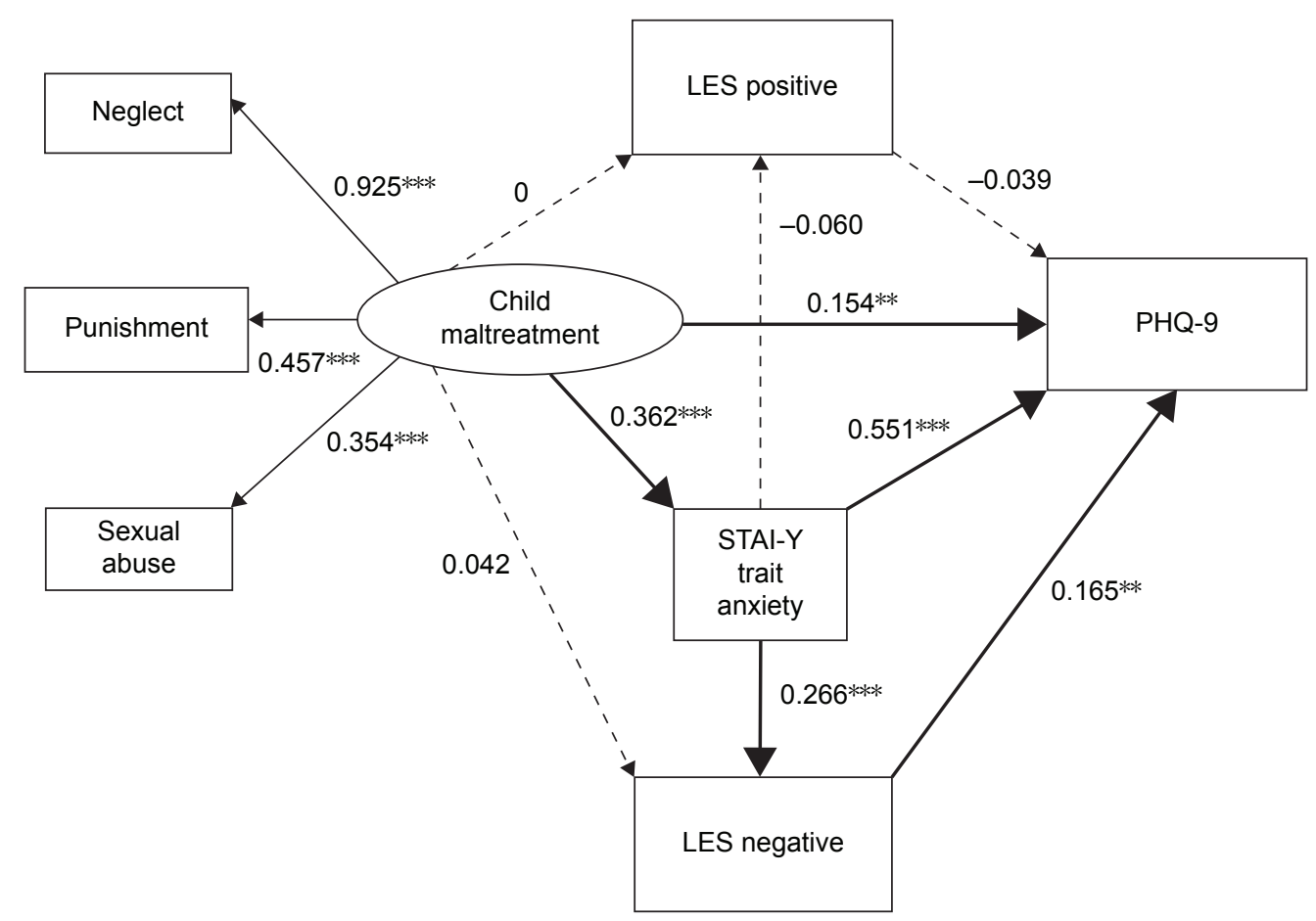

Figure I Results of covariance structure analysis in the structural equation model with child maltreatment, trait anxiety (STAI-Y trait anxiety), negative and positive appraisal of life events in adulthood (LES negative and LES positive), and depressive symptoms (PHQ-9) in 404 adult volunteer subjects from the community.

Notes: Rectangles indicate the observed variables. The oval indicates the latent variable. Arrows with solid lines represent the statistically significant pathways, and the broken lines show the nonsignificant pathways. The numbers beside the arrows show the standardized path coefficients (minimum $-\mathrm{I}$, maximum I). $* * P<0.0 \mathrm{I}$, $* * * P<0.00 \mathrm{I}$. Abbreviations: LES, Life Experiences Survey; STAI-Y, State-Trait Anxiety Inventory Form Y. 
maltreatment was considered to indirectly influence depressive symptoms and the evaluation of life events in adulthood through trait anxiety as a potential mediating factor.

The rationale of this structural equation model was based on the temporal characteristics of the questionnaires used in this study, ie, the CATS for child maltreatment evaluates adverse experiences in the childhood and teen years; STAI-Y trait anxiety evaluates the general tendency to feel anxiety as a nonpathological personal trait; the LES evaluates life events during the previous year; and the PHQ-9 evaluates depressive symptoms in the previous 2 weeks. A previous prospective, longitudinal community study showed that the average age of onset of anxiety disorders was earlier than that of depressive disorders and that most anxiety disorders were primary disorders that substantially increase the risk for secondary depression. ${ }^{25}$

In addition, this study used the latent variable "child maltreatment" instead of the CATS total score because the intention was to clarify the subtypes of child maltreatment that are associated with depressive symptoms. Furthermore, our previous studies showed that neglect, rather than punishment and sexual abuse, is the most influential factor for MDD and depressive symptoms in adult volunteers from the community. ${ }^{7,9,10}$

\section{Data analysis}

The Spearman rank correlation coefficient and the MannWhitney $U$ test (SPSS version 24, SPSS Inc., Chicago, IL, USA) were used to calculate correlations between variables and to compare variables between two categories of demographic characteristics (eg, men vs women), respectively. Furthermore, stepwise multiple regression analysis (backward elimination) using PHQ-9 as the dependent variable was performed. Demographic characteristics and scores of the questionnaire data, age, sex, current marital status, presence of offspring, living status, years of education, employment status, comorbidity of physical disease, CATS (neglect, punishment, and sexual abuse), LES change scores (positive and negative) and STAI-Y trait anxiety scores were used as independent variables.

The structural equation model was analyzed by covariance structure analysis robust maximum likelihood estimation using Mplus version 7.3 (Muthén \& Muthén, Los Angeles, CA, USA). There is no absolute standard to judge the fitness of the model by covariance structure analysis. For this reason, the model was comprehensively evaluated by combining several indices of goodness-of-fit, such as root mean square error of approximation (RMSEA), Tucker-Lewis index (TLI), and comparative fit index (CFI). A CFI $>0.95$, a TLI $>0.95$, and an RMSEA $<0.08$ indicate an acceptable fit; and a CFI $>0.97$, a TLI $>0.97$, and an RMSEA $<0.05$ indicate a good fit. ${ }^{26}$ All of the coefficients were standardized and shown (with a maximum of +1 and a minimum of -1 ) for covariance structure analysis. A $P$-value of $<0.05$ was considered to indicate a statistically significant difference between two groups.

\section{Results \\ Influence of the demographic characteristics of subjects and questionnaire data on total PHQ-9 scores}

Demographic and questionnaire data on 404 subjects and their effects on total PHQ-9 scores are shown in Table 1. Age, sex, current marital status, and the presence of offspring were significantly associated with total PHQ-9 scores. On the other hand, years of education, employment status, living status, and comorbidity of physical disease were not associated with PHQ-9 summary scores. The total CATS score, average scores of the CATS subscales (neglect, punishment, and sexual abuse), STAI-Y traits and state anxiety scores, and LES negative change score significantly correlated with PHQ-9 summary scores.

\section{Stepwise multiple regression analysis (backward elimination) of PHQ-9 summary scores}

Table 2 shows the results of stepwise multiple regression analysis (backward elimination) of PHQ-9 summary scores. We chose 14 independent factors with the PHQ-9 summary score as a dependent variable. Ten independent variables were excluded from this model. Four independent variables, namely, STAI-Y trait anxiety, CATS neglect score, LES negative change score, and age significantly predicted PHQ-9 summary scores (adjusted $R^{2}=0.466, F=82.892, P<0.001$ ). As variance inflation factors were approximately 1 , multicollinearity was denied in this multiple regression analysis.

\section{Correlation $(\rho)$ between CATS subscale scores, LES positive and negative change scores, and STAI-Y state and trait anxiety scores}

As shown in Table 1, the STAI-Y trait anxiety score, total CATS score and subscale scores, and LES negative change score were positively correlated with depressive symptoms. Furthermore, the correlation between total CATS scores 
Table I Background characteristics, PHQ summary, CATS, STAI-Y, and LES scores of the subjects, and their correlation with and effects on PHQ-9 scores

\begin{tabular}{|c|c|c|}
\hline Characteristics or measures & $\begin{array}{l}\text { Number or } \\
\text { mean } \pm \text { SD }\end{array}$ & $\begin{array}{l}\text { Correlation with PHQ-9 scores }(\rho) \text { or effect on PHQ-9 scores } \\
\text { (mean } \pm \text { SD, U-test) }\end{array}$ \\
\hline Age (years) & $42.3 \pm 11.9$ & $\rho=-0.116^{*}$ \\
\hline Sex (men:women) & $220: 184$ & Men $2.9 \pm 3.6$ vs women $3.7 \pm 4.1 *$ (U-test) \\
\hline Years of education & $15.2 \pm 2.0$ & $\rho=-0.065, P=0.193$ \\
\hline Employment status (employed:unemployed) & $341: 56$ & Employed $3.3 \pm 3.8$ vs unemployed $3.6 \pm 4.4, P=0.955$ (U-test) \\
\hline Current marital status (married:single) & 287:114 & Married $3.0 \pm 3.7$ vs single $4.0 \pm 4 . I^{* *}$ (U-test) \\
\hline Presence of offspring (yes:no) & $270: 131$ & Yes $2.9 \pm 3.6$ vs no $4.0 \pm 4.2^{* *}$ (U-test) \\
\hline Living alone (yes:no) & 101:295 & Yes $3.6 \pm 4.2$ vs no $3.1 \pm 3.7, P=0.357$ (U-test) \\
\hline Comorbidities (yes:no) & $81: 319$ & Yes $3.6 \pm 3.7$ vs no $3.2 \pm 3.8, P=0.120$ (U-test) \\
\hline PHQ summary score & $3.3 \pm 3.8$ & \\
\hline \multicolumn{3}{|l|}{ CATS (average score) } \\
\hline Sexual abuse & $0.04 \pm 0.22$ & $\rho=0.155^{* *}$ \\
\hline Neglect & $0.61 \pm 0.58$ & $\rho=0.33 \mathrm{I} * *$ \\
\hline Punishment & $1.4 I \pm 0.62$ & $\rho=0.207^{* *}$ \\
\hline Total & $0.65 \pm 0.43$ & $\rho=0.323 * *$ \\
\hline STAI-Y state anxiety & $40.3 \pm 10.3$ & $\rho=0.547^{* *}$ \\
\hline STAI-Y trait anxiety & $41.7 \pm 9.8$ & $\rho=0.624^{* *}$ \\
\hline \multicolumn{3}{|l|}{ LES (change score) } \\
\hline Negative & $1.7 \pm 3.1$ & $\rho=0.328 * *$ \\
\hline Positive & $1.7 \pm 3.0$ & $\rho=-0.054, P=0.275$ \\
\hline
\end{tabular}

Notes: Data are presented as means \pm SD or numbers. $\rho=$ Spearman rank correlation coefficient. $* P<0.05, * * P<0.01$.

Abbreviations: CATS, Child Abuse and Trauma Scale; LES, Life Experiences Survey; PHQ-9, Patient Health Questionnaire-9; STAI-Y, State-Trait Anxiety Inventory Form Y.

and subscale scores, positive and negative change scores of LES, and STAI-Y state and trait anxiety scores were analyzed (Table 3). The STAI-Y trait anxiety score significantly correlated with total CATS score and subscale scores, and positive and negative score changes of the LES, and STAI-Y state anxiety scores.

\section{Analysis of the structural equation model}

In our structural equation model, the CATS, LES, and STAI-Y trait anxiety scores affect PHQ-9 summary scores in

Table 2 Results of stepwise multiple regression analysis of PHQ-9

\begin{tabular}{l|l|l|l}
\hline $\begin{array}{l}\text { Independent factors } \\
\text { selected by stepwise } \\
\text { regression }\end{array}$ & $\begin{array}{l}\text { Standardized } \\
\text { partial regression } \\
\text { coefficient (beta) }\end{array}$ & $P$-value & VIF \\
\hline STAI-Y trait anxiety & 0.561 & $<0.001$ & 1.213 \\
CATS neglect & 0.133 & 0.001 & 1.138 \\
LES negative & 0.137 & 0.001 & 1.111 \\
Age (years) & $-0.08 \mathrm{I}$ & 0.034 & 1.019 \\
Adjusted $R^{2}=0.466$ & $F=82.892, P<0.001$ & & \\
\hline
\end{tabular}

Notes: Dependent variable: PHQ-9 summary score. The 14 independent variables tested were as follows: age, $\operatorname{sex}(\operatorname{men}=0$, women $=I)$, current marital status ( $\operatorname{single}=0$, married $=I$ ), presence of offspring (yes $=I$, no $=0$ ), living alone (yes $=0$, no=l), years of education, employment status (unemployed $=0$, employed $=I$ ), comorbidities (yes $=I$, no=0), CATS (neglect, punishment, and sexual abuse), LES change scores (positive and negative), and STAI-Y trait anxiety scores. $R^{2}=$ square sums of multiple correlation coefficient.

Abbreviations: CATS, Child Abuse and Trauma Scale; LES, Life Experiences Survey; PHQ-9, Patient Health Questionnaire-9; STAI-Y, State-Trait Anxiety Inventory Form Y; VIF, variance inflation factor. a complex manner, and this model was analyzed by covariance structure analysis. We treated scores of the three CATS subscales (sexual abuse, neglect, and punishment) as the observed variables, and "child maltreatment" as the latent variable (Figure 1). The goodness-of-fit of this structural equation model was good $\left(\chi^{2}\right.$ statistic $P=0.445$, CFI $=1.000$, TLI $=1.001$, and RMSEA $=0.000)$. This model explained $47.8 \%$ of the variability in depressive symptoms $\left(R^{2}=0.478\right)$.

\section{Direct effects}

Child maltreatment had significant positive effects on PHQ-9 scores (severity of depressive symptoms) and STAI-Y trait anxiety scores. However, child maltreatment had no significant effect on LES negative and positive change scores. STAI-Y trait anxiety scores had significant positive effects on LES negative change scores and PHQ-9 scores, but not on LES positive change scores. Negative change scores of LES increased depressive symptoms, but positive change scores of LES did not affect depressive symptoms. Among the subscales of the CATS, the path coefficient from "child maltreatment" to neglect subscale scores was largest.

\section{Indirect effects}

Child maltreatment indirectly increased PHQ-9 scores through increasing STAI-Y trait anxiety scores (standardized 
Table 3 Correlation $(\rho)$ between subscale scores of the CATS, the LES positive and negative scores, and the STAI-Y state and trait scores

\begin{tabular}{l|l|l|l|l|l|l|l|l}
\hline Measures & CATS Neg & CATS Pun & CATS Sex & CATS total & LES positive & LES negative & STAI-Y state & STAI-Y trait \\
\hline CATS Neg & 1.000 & $0.309 * *$ & $0.218^{* *}$ & $0.862^{* *}$ & -0.029 & $0.148^{* *}$ & $0.218^{* *}$ & $0.312^{* *}$ \\
CATS Pun & & 1.000 & $0.156^{* *}$ & $0.609 * *$ & -0.065 & 0.073 & 0.094 & $0.189^{* *}$ \\
CATS Sex & & & 1.000 & $0.262^{* *}$ & -0.025 & 0.028 & 0.065 & $0.115^{*}$ \\
CATS total & & & & 1.000 & -0.022 & $0.128^{*}$ & $0.200^{* *}$ & $0.296^{* *}$ \\
LES positive & & & & & 1.000 & $0.122^{*}$ & $-0.143^{* *}$ & $-0.101^{*}$ \\
LES negative & & & & & & 1.000 & $0.208^{* *}$ & $0.266^{* *}$ \\
STAI-Y state & & & & & & & 1.000 & $0.739 * *$ \\
STAI-Y trait & & & & & & & & \\
\hline
\end{tabular}

Notes: $\rho=$ Spearman rank correlation coefficient; $* P<0.05, * * P<0.0$ I.

Abbreviations: CATS, Child Abuse and Trauma Scale; LES, Life Experiences Survey; Neg, neglect subscale; Pun, punishment subscale; STAI-Y, State-Trait Anxiety Inventory Form Y; Sex, sexual abuse subscale.

coefficient $0.199, P<0.001$ ). Child maltreatment indirectly increased negative change scores of the LES through increasing the STAI-Y trait anxiety score (standardized coefficient 0.096, $P<0.001)$. Furthermore, the STAI-Y trait anxiety score indirectly increased PHQ-9 via a positive effect on negative change scores of the LES (standardized coefficient $0.044, P<0.05)$. Finally, child maltreatment indirectly and significantly increased the PHQ-9 summary score (standardized coefficient $0.016, P<0.05$ ) via a positive effect on the two combined paths of STAI-Y trait anxiety and LES negative change scores. However, no indirect effect via the positive change score of the LES was observed in this model.

\section{Discussion}

To our knowledge, this is the first study demonstrating that the experience of child maltreatment indirectly affects depressive symptoms through enhanced trait anxiety, a potential mediator, as well as having a direct effect. In this study, the experience of child maltreatment, trait anxiety, and negative appraisal of life events directly influenced depressive symptoms in 404 volunteer adult subjects from the community. These results suggest that trait anxiety may be a mediator for the effects of child maltreatment on depressive symptoms and the negative appraisal of life events, but not the positive appraisal of life events.

Several studies reported that experiencing child maltreatment, such as physical and sexual abuse, and total adverse childhood experiences increased trait anxiety in university students and patients with anxiety disorder. ${ }^{27,28}$ Moreover, we reported that childhood parental bonding affected adulthood trait anxiety indirectly through self-esteem; trait anxiety was decreased by high parental care and increased by parental overprotection, respectively. ${ }^{14}$ The results of earlier studies agree with the results of the present study, indicating a close association between adverse childhood experiences and trait anxiety, which is a characteristic of vulnerability and a risk factor of depression. ${ }^{16,17,29}$

Neuroticism, which is a personality trait, has been established as a risk factor for developing major depression. High neuroticism tends to induce the development of depressive episodes in response to stressful life events. ${ }^{5}$ Our previous studies suggested that neuroticism is a mediator for the effects of the experience of child maltreatment and quality of parenting on depressive symptoms in adulthood and the negative appraisal of life events. ${ }^{12,13}$ Neuroticism shares some characteristics with trait anxiety ${ }^{29}$ and both are moderately and positively associated. ${ }^{30}$ As in the case of neuroticism, individuals with a high anxiety trait show hyper responsiveness to threatening stimuli, as well as attentional biases that facilitate the detection of threats and aversiveness. ${ }^{31}$ Negative life event appraisals were significantly associated with trait anxiety in this study and in the original study that developed the LES, whereas positive life event appraisals were not. ${ }^{20}$ The SEM results of our present study suggest that trait anxiety increases the vulnerability to stress, leading to more severe depressive symptoms.

In the present study, among the subscales of child maltreatment, a major role of neglect on depressive symptoms in adulthood was found compared with sexual abuse and punishment. In the results of multiple regression analysis of PHQ-9 summary scores in this study, neglect on CATS, but not sexual abuse or punishment, was a significant predictor of depressive symptoms. The coefficient of neglect from the latent variable of "child maltreatment" in the SEM was the largest among the three subscales of the CATS, consistent with our previous studies. ${ }^{7,13}$ Similarly, a high neglect score on the CATS significantly predicted an MDD diagnosis in the comparison between healthy controls and patients with MDD. ${ }^{9}$ Because our findings were obtained from studies using the CATS and Japanese subjects, measurement 
invariance should be confirmed. However, several studies using a different abuse questionnaire (the Childhood Trauma Questionnaire) and subjects of different countries also demonstrated that emotional abuse and neglect have a closer association with depressive symptoms than other types of abuse. ${ }^{32,33}$ Hence, neglect is considered to be the most influential factor for depressive symptoms among several types of child maltreatment.

Interestingly, trait anxiety, negative life event appraisal, and their combination may play a mediating role in the effects of the experience of child maltreatment on depressive symptoms. In this study, the experience of child maltreatment showed a significant indirect effect on adulthood depressive symptoms through pathways including increased trait anxiety, increased negative life event appraisals, and both, which might reflect vulnerability to stress. A similar phenomenon was reported for neuroticism. ${ }^{13}$ Consistent with our present and previous studies, other researchers reported that an increased exposure to negative life events in adulthood partly mediates the association between childhood adversities and depression in adulthood. ${ }^{34}$

The experience of child maltreatment induces long-lived hyperactivity of corticotropin-releasing factor systems or the hypothalamic-pituitary-adrenal (HPA) axis and hypofunction of the hippocampus. ${ }^{35,36}$ Interestingly, hyperactivity of the HPA axis is also reported in increased trait anxiety and may be a vulnerability factor for stress-induced depression. ${ }^{16,31}$ Amygdalar activation analyzed by functional MRI is observed in both anxiety disorders and major depression ${ }^{37,38}$ and is increased by the experience of child maltreatment and trait anxiety. ${ }^{39,40}$ These biological changes associated with child maltreatment and increased trait anxiety are suggested to be the neural basis for the onset of depression.

The experience of child maltreatment is an etiological factor for MDD. However, how child maltreatment causes MDD is unknown. Our model suggests that high trait anxiety induced by the experience of child maltreatment may increase sensitivity to stress, leading to the onset of depression. Consistent with this idea, child maltreatment is associated with the comorbidity of anxiety disorders in major depression, ${ }^{41}$ and most anxiety disorders are primary disorders that substantially increase the risk for secondary depression. ${ }^{25}$ Clinically, the psychological and social intervention for reducing trait anxiety as well as reducing experiences of child maltreatment may prevent major depression. This study proposes a clinical suggestion that we should positively evaluate the combination of child maltreatment and trait anxiety in depressive patients.
There are some limitations to this study. The first is that the subjects of this study are volunteer subjects from the community including healthy people, and hence the results may not be generalizable to MDD patients. The second limitation is that this study used self-written questionnaires that rely on the subjects' memories. The CATS used to measure child maltreatment is a questionnaire in which subjects retrospectively recall experiences in childhood, and hence may be affected by recall bias. The third limitation is that because this study is a cross-sectional study, the causality between the experience of child maltreatment and depression is not clear. Santor et $\mathrm{al}^{42}$ showed that a personality trait was relatively stable and largely influenced by the baseline trait, but a small change in a personality trait was caused by depressive symptoms. Therefore, there is the possibility that reverse causality may be established from our data but the reversed effect might be relatively small. The fourth limitation is that $47.4 \%$ of volunteers agreed to participate in this study and provided a complete response, which might lead to selection bias. The fifth limitation is that we did not investigate the association of medications or particular comorbidities.

\section{Conclusion}

The present study indicates that trait anxiety may play a mediating role in the effects of the experience of child maltreatment on depressive symptoms and the negative appraisal of life events in adulthood. Longitudinal studies are necessary to clarify temporal changes in the associations observed between these variables. These findings suggest the close association between anxiety and depression and suggest that interventions to reduce cases of child maltreatment will decrease the incidence of trait anxiety in adulthood, leading to the prevention of MDD and depressive symptoms.

\section{Acknowledgments}

This work was partly supported by a Grant-in-Aid for Scientific Research (no 16K10194, to T. Inoue) from the Japanese Ministry of Education, Culture, Sports, Science and Technology, the Research and Development Grants for Comprehensive Research for Persons with Disabilities from the Japan Agency for Medical Research and Development under grant no JP18dk0307060 (to T Inoue), and SENSHIN Medical Research Foundation (to T Inoue).

\section{Author contributions}

TI designed the study and wrote the protocol. YU and TI collected and analyzed the data. All authors contributed to data analysis and drafting and critically revising the manuscript. 
In addition, all authors gave final approval for the present version of the manuscript to be published and agreed to be accountable for all aspects of the work.

\section{Disclosure}

JM has received personal compensation from Otsuka Pharmaceutical, Eli Lilly, Astellas, and Meiji Yasuda Mental Health Foundation and grants from Pfizer. MI has received personal compensation from Otsuka Pharmaceutical, Pfizer, Eli Lilly, Mitsubishi Tanabe Pharma, Mochida Pharmaceutical, Meiji Seika Pharma, Janssen Pharmaceutical, Takeda Pharmaceutical Company, MSD, Dainippon Sumitomo Pharma, and Eisai; grants from Otsuka Pharmaceutical, Eli Lilly, Eisai, Shionogi, Takeda Pharmaceutical, MSD, and Pfizer; and is a member of the advisory board of Meiji Seika Pharma. IK has received personal compensation from Astellas, Chugai Pharmaceutical, Daiichi Sankyo, Dainippon Sumitomo Pharma, Eisai, Eli Lilly, Janssen Pharmaceutical, Kyowa Hakko Kirin, Meiji Seika Pharma, MSD, Nippon Chemiphar, Novartis Pharma, Ono Pharmaceutical, Otsuka Pharmaceutical, Pfizer, Tanabe Mitsubishi Pharma, Shionogi, and Yoshitomiyakuhin; and has received research grants from AbbVie GK, Asahi Kasei Pharma, Astellas, Boehringer Ingelheim, Chugai Pharmaceutical, Daiichi Sankyo, Dainippon Sumitomo Pharma, Eisai, Eli Lilly, GlaxoSmithKline, Kyowa Hakko Kirin, Meiji Seika Pharma, MSD, Novartis Pharma, Ono Pharmaceutical, Otsuka Pharmaceutical, Pfizer, Takeda Pharmaceutical, Tanabe Mitsubishi Pharma, Shionogi, and Yoshitomiyakuhin; and is a member of the advisory board of Dainippon Sumitomo Pharma and Tanabe Mitsubishi Pharma. TI has received personal compensation from GlaxoSmithKline, Mochida Pharmaceutical, Asahi Kasei Pharma, and Shionogi; grants from Astellas; and grants and personal compensation from Otsuka Pharmaceutical, Dainippon Sumitomo Pharma, Eli Lilly, Eisai, Mitsubishi Tanabe Pharma, Pfizer, AbbVie GK, MSD, Yoshitomiyakuhin, Takeda Pharmaceutical, and Meiji Seika Pharma; and is a member of the advisory boards of GlaxoSmithKline, Pfizer, Eli Lilly, Mochida Pharmaceutical, and Mitsubishi Tanabe Pharma. The authors report no other conflicts of interest in this work.

\section{References}

1. Sadock B, Sadock V, Kaplan RP. Kaplan \& Sadock's Synopsis of Psychiatry: Behavioral Sciences/Clinical Psychiatry. 11th ed. Philadelphia: Lippincott Williams \& Wilkins, Inc; 2014.

2. Global Burden of Disease Study 2013 Collaborators. Global, regional, and national incidence, prevalence, and years lived with disability for 301 acute and chronic diseases and injuries in 188 countries, 1990-2013: a systematic analysis for the Global Burden of Disease Study 2013. Lancet. 2015;386(9995):743-800.
3. Alloy LB, Abramson LY, Smith JM, Gibb BE, Neeren AM. Role of parenting and maltreatment histories in unipolar and bipolar mood disorders: mediation by cognitive vulnerability to depression. Clin Child Fam Psychol Rev. 2006;9(1):23-64.

4. Caspi A, Sugden K, Moffitt TE, et al. Influence of life stress on depression: moderation by a polymorphism in the 5-HTT gene. Science. 2003; 301(5631):386-389.

5. Kendler KS, Kuhn J, Prescott CA. The interrelationship of neuroticism, sex, and stressful life events in the prediction of episodes of major depression. Am J Psychiatry. 2004;161(4):631-636.

6. Kessler RC, Magee WJ. Childhood adversities and adult depression: basic patterns of association in a US national survey. Psychol Med. 1993; 23(3):679-690.

7. Nakai $\mathrm{Y}$, Inoue T, Toda $\mathrm{H}$, et al. The influence of childhood abuse, adult stressful life events and temperaments on depressive symptoms in the nonclinical general adult population. J Affect Disord. 2014;158: 101-107.

8. Nakai $\mathrm{Y}$, Inoue $\mathrm{T}$, Chen $\mathrm{C}$, et al. The moderator effects of affective temperaments, childhood abuse and adult stressful life events on depressive symptoms in the nonclinical general adult population. $J$ Affect Disord. 2015;187:203-210.

9. Toda H, Inoue T, Tsunoda T, et al. The structural equation analysis of childhood abuse, adult stressful life events, and temperaments in major depressive disorders and their influence on refractoriness. Neuropsychiatr Dis Treat. 2015;11:2079-2090.

10. Toda $\mathrm{H}$, Inoue $\mathrm{T}$, Tsunoda $\mathrm{T}$, et al. Affective temperaments play an important role in the relationship between childhood abuse and depressive symptoms in major depressive disorder. Psychiatry Res. 2016;236: 142-147.

11. Enns MW, Cox BJ, Larsen DK. Perceptions of parental bonding and symptom severity in adults with depression: mediation by personality dimensions. Can J Psychiatry. 2000;45(3):263-268.

12. Ono Y, Takaesu Y, Nakai Y, et al. The influence of parental care and overprotection, neuroticism and adult stressful life events on depressive symptoms in the general adult population. $J$ Affect Disord. 2017;217: 66-72.

13. Ono K, Takaesu Y, Nakai Y, et al. Associations among depressive symptoms, childhood abuse, neuroticism, and adult stressful life events in the general adult population. Neuropsychiatr Dis Treat. 2017;13: $477-482$.

14. Shimura A, Takaesu Y, Nakai Y, et al. Childhood parental bonding affects adulthood trait anxiety through self-esteem. Compr Psychiatry. 2017; 74:15-20.

15. Min JA, Lee NB, Lee CU, Lee C, Chae JH. Low trait anxiety, high resilience, and their interaction as possible predictors for treatment response in patients with depression. J Affect Disord. 2012;137(1-3):61-69.

16. Sandi C, Richter-Levin G. From high anxiety trait to depression: a neurocognitive hypothesis. Trends Neurosci. 2009;32(6):312-320.

17. Kok L, Sep MS, Veldhuijzen DS, et al. Trait anxiety mediates the effect of stress exposure on post-traumatic stress disorder and depression risk in cardiac surgery patients. J Affect Disord. 2016;206:216-223.

18. Spielberger CD. Manual for the State-Trait Anxiety Inventory STAI (form Y). Palo Alto: Consulting Psychologists Press; 1983.

19. Sanders B, Becker-Lausen E. The measurement of psychological maltreatment: early data on the child abuse and trauma scale. Child Abuse Negl. 1995;19(3):315-323.

20. Sarason IG, Johnson JH, Siegel JM. Assessing the impact of life changes: development of the life experiences survey. $J$ Consult Clin Psychol. 1978;46(5):932-946.

21. Spitzer RL, Kroenke K, Williams JB. Validation and utility of a selfreport version of PRIME-MD: the PHQ primary care study. Primary Care Evaluation of Mental Disorders. Patient Health Questionnaire. JAMA. 1999;282(18):1737-1744.

22. Muramatsu K, Miyaoka H, Kamijima K, et al. The patient health questionnaire, Japanese version: validity according to the mini-international neuropsychiatric interview-plus. Psychol Rep. 2007;101(3 Pt 1): 952-960. 
23. Inoue $\mathrm{T}$, Tanaka $\mathrm{T}, \mathrm{Nakagawa} \mathrm{S}$, et al. Utility and limitations of PHQ-9 in a clinic specializing in psychiatric care. BMC Psychiatry. 2012; 12:73.

24. Tanabe H, Ozawa S, Goto K. Psychometric properties of the Japanese version of the Child Abuse and Trauma Scale (CATS). The 9th Annual Meeting of the Japanese Society for Traumatic Stress Studies; Kobe. Japanese. March 6, 2010.

25. Wittchen HU, Kessler RC, Pfister H, Lieb M. Why do people with anxiety disorders become depressed? A prospective-longitudinal community study. Acta Psychiatr Scand Suppl. 2000;(406):14-23.

26. Schermelleh-Engel K, Moosbrugger H, Müller H. Evaluating the fit of structural equation models: tests of significance and descriptive goodness-of-fit measures. MPR online. 2003;8(2):23-74.

27. Mancini C, van Ameringen M, Macmillan H. Relationship of childhood sexual and physical abuse to anxiety disorders. J Nerv Ment Dis. 1995;183(5):309-314.

28. Reiser SJ, Mcmillan KA, Wright KD, Asmundson GJ. Adverse childhood experiences and health anxiety in adulthood. Child Abuse Negl. 2014;38(3):407-413.

29. Bishop S, Forster S. Trait anxiety, neuroticism, and the brain basis of vulnerability to affective disorder. In: Armony J, Vuilleumier P, editors. The Cambridge Handbook of Human Affective Neuroscience. Cambridge: Cambridge University Press; 2013:553-574.

30. Gonda X, Fountoulakis KN, Juhasz G, et al. Association of the s allele of the 5-HTTLPR with neuroticism-related traits and temperaments in a psychiatrically healthy population. Eur Arch Psychiatry Clin Neurosci. 2009;259(2):106-113.

31. Weger M, Sandi C. High anxiety trait: a vulnerable phenotype for stress-induced depression. Neurosci Biobehav Rev. 2018;87:27-37.

32. Gibb BE, Chelminski I, Zimmerman M. Childhood emotional, physical, and sexual abuse, and diagnoses of depressive and anxiety disorders in adult psychiatric outpatients. Depress Anxiety. 2007;24(4):256-263.
33. Lee SW, Bae GY, Rim HD, et al. Mediating effect of resilience on the association between emotional neglect and depressive symptoms. Psychiatry Investig. 2018;15(1):62-69.

34. Korkeila J, Vahtera J, Nabi H, et al. Childhood adversities, adulthood life events and depression. J Affect Disord. 2010;127(1-3):130-138.

35. Frodl T, O'Keane V. How does the brain deal with cumulative stress? A review with focus on developmental stress, HPA axis function and hippocampal structure in humans. Neurobiol Dis. 2013;52:24-37.

36. Heim $\mathrm{C}$, Nemeroff $\mathrm{CB}$. The role of childhood trauma in the neurobiology of mood and anxiety disorders: preclinical and clinical studies. Biol Psychiatry. 2001;49(12):1023-1039.

37. Sheline YI, Barch DM, Donnelly JM, Ollinger JM, Snyder AZ, Mintun MA. Increased amygdala response to masked emotional faces in depressed subjects resolves with antidepressant treatment: an fMRI study. Biol Psychiatry. 2001;50(9):651-658.

38. Gingnell M, Frick A, Engman J, et al. Combining escitalopram and cognitive-behavioural therapy for social anxiety disorder: randomised controlled fMRI trial. Br J Psychiatry. 2016;209(3):229-235.

39. Indovina I, Robbins TW, Núñez-Elizalde AO, Dunn BD, Bishop SJ. Fear-conditioning mechanisms associated with trait vulnerability to anxiety in humans. Neuron. 2011;69(3):563-571.

40. van Harmelen AL, van Tol MJ, Demenescu LR, et al. Enhanced amygdala reactivity to emotional faces in adults reporting childhood emotional maltreatment. Soc Cogn Affect Neurosci. 2013;8(4):362-369.

41. Cyranowski JM, Schott LL, Kravitz HM, et al. Psychosocial features associated with lifetime comorbidity of major depression and anxiety disorders among a community sample of mid-life women: the SWAN mental health study. Depress Anxiety. 2012;29(12):1050-1057.

42. Santor DA, Bagby RM, Joffe RT. Evaluating stability and change in personality and depression. J Pers Soc Psychol. 1997;73(6):1354-1362.
Neuropsychiatric Disease and Treatment

\section{Publish your work in this journal}

Neuropsychiatric Disease and Treatment is an international, peerreviewed journal of clinical therapeutics and pharmacology focusing on concise rapid reporting of clinical or pre-clinical studies on a range of neuropsychiatric and neurological disorders. This journal is indexed on PubMed Central, the 'PsycINFO' database and CAS,

\section{Dovepress}

and is the official journal of The International Neuropsychiatric Association (INA). The manuscript management system is completely online and includes a very quick and fair peer-review system, which is all easy to use. Visit http://www.dovepress.com/testimonials.php to read real quotes from published authors. 